\title{
Epidemiología de la enfermedad fúngica invasora por hongos filamentosos en el período 2005 a 2015, en un hospital universitario en Santiago, Chile
}

\author{
Pablo Valenzuela ${ }^{1}$, Paulette Legarraga ${ }^{2}$ y Ricardo Rabagliati ${ }^{1}$
}

\footnotetext{
'Departamento de Enfermedades

Infecciosas del Adulto. Escuela de Medicina. Pontificia Universidad Católica de Chile. Santiago, Chile. ${ }^{2}$ Departamento de Laboratorios Clínicos. Escuela de Medicina. Pontificia Universidad Católica de Chile. Santiago, Chile.

Los autores declaran la ausencia de conflictos de interés. No hubo financiamiento externo a los autores.

Recibido: 5 de agosto de 2019 Aceptado: 10 de noviembre de

Correspondencia a: Ricardo Rabagliati Borie rabagli@med.puc.cl
}

\begin{abstract}
Epidemiology of invasive fungal disease by filamentous fungi in the period 2005 to 2015, in a university hospital in Santiago, Chile

Background: Invasive fungal disease (IFD) due to filamentous fungi is increasingly common. Aim: To study the epidemiology of EFI in hospitalized adults in our center. Methods: Retrospective study of adult patients of a university hospital in Santiago, Chile, with EFI due to filamentous fungi between January 2005 and December 2015. Results: 125 episodes were identified, being $48 \%$ proven, $40 \%$ probable and $11 \%$ possible according to EORTC/MSG criteria, overall incidence was $0.47 / 1,000$ admissions, $57 \%$ male patients and age $50 \pm 16$ years. $66.4 \%$ had hematological pathology, $11.2 \%$ solid organ transplant, $11.2 \%$ rheumatology diseases, $11.2 \%$ other conditions. The risk factors were neutropenia $44 \%$, corticosteroid therapy $21 \%$, immunosuppressants $13 \%$. The most frequent mould identified were Aspergillus spp (53.6\%), Mucorales (16\%), Fusarium spp (8.8\%), Alternaria spp (5.6\%) and other filamentous (3.2\%). All received antifungals, $82 \%$ monotherapy, 18\% combined therapy, there was surgical defocation in $90 \%$ of mucormycosis. The overall mortality was $42 \%$. When comparing 2005 2009 vs 2010-2015, there was a significant increase in incidence and a tendency to lower mortality in the second period. Conclusions: Over a period of 10 years, we observed an increase in the incidence of EFI by filamentous, aspergillosis was the most frequent etiology and the overall mortality was $42 \%$.

Keywords: Moulds; invasive fungal disease; epidemiology.

Palabras clave: Hongos filamentosos; enfermedad fúngica invasora; epidemiología.
\end{abstract}

\section{Introducción}

L a enfermedad fúngica invasora (EFI) por hongos filamentosos incluye infecciones producidas por Aspergillus spp., agentes de mucormicosis (Rhizopus, Mucor, Rhizomucor, Lichtheimia, Cunninghamella, Apophysomyces, Sakasenea, Syncephalastrum racemosum), hialohifomicosis (Fusarium, Scedosporium, Paecilomyces, Acremonium, Schizophyllum, Rasamsonia) y feohifomicosis (Alternaria, Bipolaris, Cladophialophora, Exophiala), que agregan morbilidad a pacientes intensamente inmunocomprometidos, generando alta mortalidad y elevación de los costos en su manejo por consumo de antifúngicos, eventuales cirugías y prolongación de estadías hospitalaria ${ }^{1-6}$. Los cambios demográficos de la población a nivel global, con incremento de la expectativa de vida, el aumento de enfermedades crónicas como cáncer, diabetes mellitus, requerimientos de trasplantes de precursores hematopoyéticos (TPH) y órganos sólidos (TOS), enfermedades inflamatorias y otras condiciones que requieren manejo inmunosupresor, han aumentado la población susceptible a este tipo de infecciones, a lo que se ha sumado durante el último tiempo la descripción de casos de EFI por filamentosos en pacientes aparentemente inmunocompetentes, tales como casos de aspergilosis en pacientes críticos, post-influenza, falla hepática aguda y terapia de reemplazo renal', así como también brotes de EFI por filamentosos asociados a cirugías ${ }^{8}$ o contaminación de fármacos ${ }^{9,10}$. Las nuevas técnicas diagnósticas basadas en biomarcadores como galactomanana (GM), $\beta$-D-glucano (BDG), reacción de polimerasa en cadena (RPC), interpretación de imágenes radiológicas y estudio histopatológico, han permitido documentar cada vez mejor los diagnósticos sistematizados con los criterios de consenso de la EORTC/MSG ${ }^{11}$.

En Chile, hay poca literatura científica sobre la epidemiología de la EFI por hongos filamentosos en pacientes adultos. Un estudio de estimación de carga de enfermedad fúngica a nivel nacional, planteó que la frecuencia de EFI por Aspergillus spp. era de 1,7/100.000 individuos y por agentes de mucormicosis de $0,2 / 100.000^{12}$. Un estudio retrospectivo de nuestro centro, que incluyó la descripción de EFI en pacientes hematológicos y TPH, entre 2004 y 2008, describió 41 episodios de EFI, identificando que la 
mayor frecuencia era por Aspergillus spp., luego Mucorales, seguido de Fusarium spp. ${ }^{13}$ Cruz y cols. publicaron un estudio prospectivo de cinco centros en Valparaíso entre 2004 y 2009, con 15 episodios de infecciones por hongos filamentosos, correspondiendo mayoritariamente a aspergilosis $^{14}$. En un segundo estudio publicaron 18 casos de EFI cuya identificación se basó mayoritariamente en GM e identificación por cultivos de dos episodios causados por Rhizopus y Sarocladium ${ }^{15}$. Otro dato de interés de la frecuencia de EFI por filamentosos en nuestro país es el de un estudio prospectivo de la etiología de 105 episodios de neutropenia febril (NF) en leucemia y linfoma, en un hospital público y otro privado de Santiago, que evidenció $20 \%$ de EFI, siendo el $80 \%$ atribuible a aspergilosis ${ }^{16}$.

La importancia de conocer la epidemiologia de la EFI por filamentosos es contribuir al mejor enfrentamiento del problema tanto de la perspectiva de la prevención, el diagnóstico y tratamiento. Sin duda, el contar con datos locales permite sensibilizar a infectólogos, así como a diferentes especialistas hemato-oncólogos, especialistas en trasplantes, reumatólogos, internistas e intensivistas para tener un elevada sospecha a este tipo de infecciones, introducir protocolos diagnósticos que incluyan todos los elementos disponibles en la actualidad como microbiología básica y molecular, biomarcadores, imagenología y estudio histopatológico, así como disponer del arsenal terapéutico necesario que incluya los antifúngicos recomendados y políticas de prevención focalizadas a grupos de pacientes de mayor riesgo.

Por este motivo, nos planteamos estudiar la epidemiología de las EFI causadas por hongos filamentosos en adultos a traves de una revisión de casos en nuestro centro, tanto en pacientes hemato-oncológicos como portadores de otras condiciones de base, a fin de comprender mejor las características de los pacientes afectados por este tipo de infecciones y confirmar las etiologías más frecuentemente descritas en estudios previos.

\section{Pacientes y Métodos}

Estudio retrospectivo de casos de EFI por hongos filamentosos, en pacientes mayores de 18 años, diagnosticados entre el 1 de enero del año 2005 y 31 de diciembre de 2015 internados en el Hospital Clínico y la Clínica UC de la Red de Salud UC-CHRISTUS.

Este centro académico asistencial corresponde a un centro de nivel terciario de atención, localizado en la ciudad de Santiago, cuenta con 468 camas, 368 $(78,6 \%)$ para atención de pacientes adultos con patología médico-quirúrgica, de las cuales $11 \%$ son para pacientes hematológicos u oncológicos para administración de quimioterapia o manejo de sus complicaciones y $27,4 \%$ de las camas corresponden a unidades de intermedio o intensivo para pacientes adultos con diversas condiciones de mayor complejidad, incluyendo patología oncológica, hematológica, TPH y TOS, además de otras condiciones médicas y quirúrgicas. Anualmente, en nuestro centro se tratan aproximadamente 80 casos de pacientes adultos con leucemias agudas, se realizan $60 \mathrm{TPH}(50 \%$ alogénicos $)$ y hasta 74 casos anuales de TOS, incluyendo en frecuencia hígado, riñón, corazón, riñón-páncreas e intestino.

Para el propósito del estudio se revisaron los registros del laboratorio de microbiología de cultivos positivos de hongos filamentosos o marcadores positivos (GM y BDG) complementado con los datos del servicio de anatomía patológica de registros de observación de hongos filamentosos en las muestras analizadas durante el período del estudio.

Se procedió a revisar las fichas clínicas de los pacientes, registrando los datos de interés como edad, sexo, factores de riesgo de EFI del hospedero, co-morbilidades, manifestaciones clínicas, resultados de exámenes de laboratorio como recuento absoluto de neutrófilos, índice óptico (IO) de GM sérico y en lavado bronco-alveolar (LBA), tinciones de Gram, calcoflúor, cultivos corriente y de hongos obtenidos de líquido estéril y/o tejido, estudios de imágenes, etiología de la infección fúngica, fecha del episodio, clasificación diagnóstica de la EFI según los criterios EORTG/MSG en infección probada, probable y posible (Tabla 1$)^{11}$, terapia antifúngica, concentraciones

\begin{tabular}{|c|c|}
\hline Hongo filamentoso & n $\quad(\%)$ \\
\hline $\begin{array}{l}\text { Aspergillus spp. } \\
\text { - A. fumigatus } \\
\text { - A. flavus } \\
\text { - A. terreus } \\
\text { - A. niger } \\
\text { - Aspergillus spp. }\end{array}$ & $\begin{aligned} 67 & (53,6) \\
28 & (22,4) \\
5 & (4) \\
5 & (4) \\
2 & (1,6) \\
27 & (21,6)\end{aligned}$ \\
\hline $\begin{array}{l}\text { Mucormicosis } \\
\text { - Rhizopus spp } \\
\text { - Mucor spp }\end{array}$ & $\begin{array}{l}20(16) \\
13(10,4) \\
7 \quad(5,6)\end{array}$ \\
\hline $\begin{array}{l}\text { Fusarium spp. } \\
\text { - F. solani } \\
\text { - Fusarium sp. }\end{array}$ & $\begin{aligned} 11 & (8,8) \\
2 & (1,6) \\
9 & (7,2)\end{aligned}$ \\
\hline Alternaria spp. & $7 \quad(5,6)$ \\
\hline Penicillium spp. & $1 \quad(0,8)$ \\
\hline Apiospermium spp. & $1 \quad(0,8)$ \\
\hline Paecilomyces spp. & $1 \quad(0,8)$ \\
\hline Chrysonilia spp. & $1(0,8)$ \\
\hline Cultivo negativo & $16(12,8)$ \\
\hline
\end{tabular}


plasmáticas de voriconazol, tiempo de hospitalización y mortalidad a los 30 días del diagnóstico, considerando mortalidad atribuible a la EFI si este episodio fue el responsable de la descompensación final y muerte del paciente según los registros de la ficha clínica.

Los datos fueron recolectados en una ficha diseñada para el estudio y luego se generó una base de datos en programa Microsoft Excel ${ }^{\circledR}$. Los resultados de las variables nominales se expresaron en porcentajes y de las variables continuas en promedio \pm desviación estándar. El análisis estadístico se realizó con el programa Stata versión 12, para el análisis de los datos se utilizó las pruebas de $\chi^{2} \mathrm{o}$ Exacta de Fisher para variables cualitativas, $t$-student para

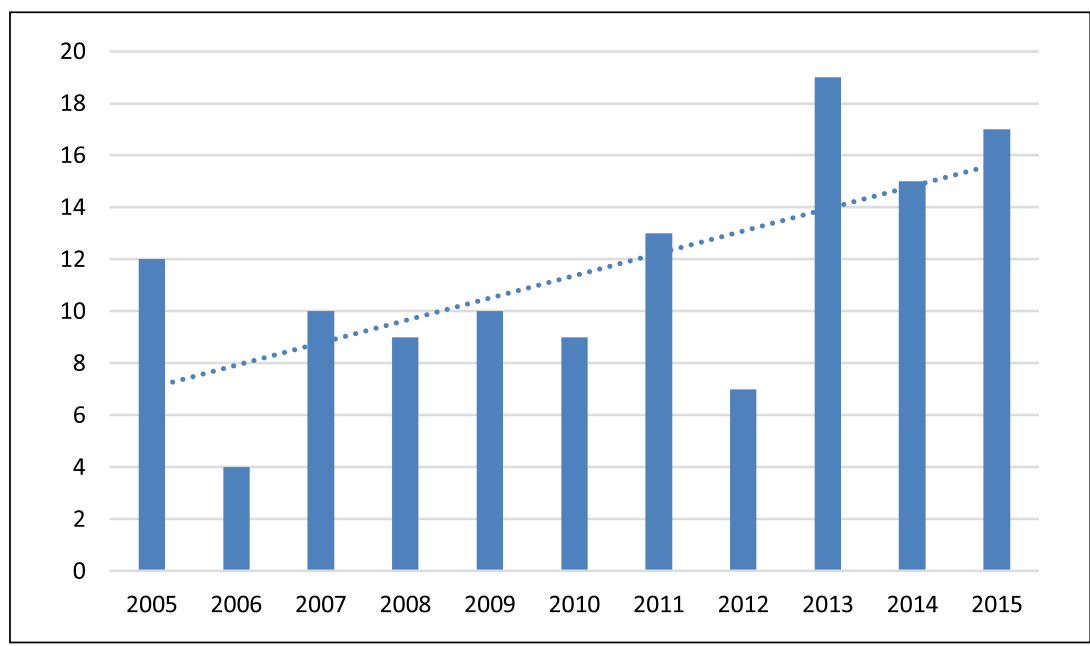

Figura 1A. Número de episodios anuales para 125 episodios de EFI por hongos filamentosos en el período 2005-2015. La línea de tendencia muestra el aumento progresivo de casos.

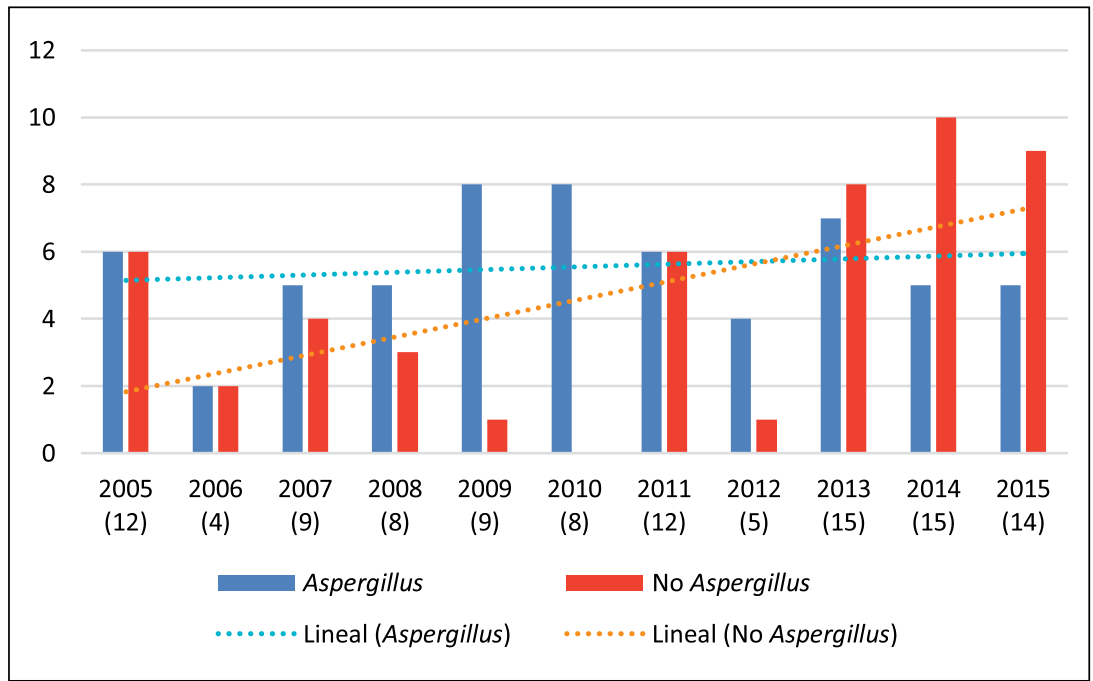

Figura 1B. Número de episodios anuales para 111 casos probados y probables de aspergilosis y noaspergilosis. La línea de tendencia muestra que el incremento es mayor para el grupo de filamentosos correspondientes a no-aspergilosis. variables continuas, test de proporciones para comparar tasas y análisis de varianza para comparar más de dos promedios, considerándose una diferencia estadísticamente significativa valor $\mathrm{p}<0,05$. La incidencia de la EFI se calculó utilizando como denominador el número de egresos hospitalarios de pacientes adultos en el período de estudio.

Este trabajo contó con la aprobación del Comité de Ética de Investigación de la Facultad de Medicina de la Pontificia Universidad Católica de Chile (Proyecto 16043, aprobado el 5 de abril de 2016).

\section{Resultados}

Se identificaron 125 episodios de EFI por hongos filamentosos en 125 pacientes adultos, correspondiendo a una incidencia global de 0,47 por 1.000 egresos (125 episodios/268.188 egresos), observándose una tendencia al incremento del número de casos diagnosticados por año desde 2005 al 2015 (Figura 1A); destaca una tendencia de incremento aún mayor para el grupo de hongos filamentosos no-Aspergillus (Figura 1B). Del total de episodios de EFI, las etiologías fueron 67 (54\%) aspergilosis correspondiendo a una incidencia de 0,25 por 1.000 egresos, 20 $(16 \%)$ mucormicosis equivalente a incidencia de 0,07 por 1.000 egresos, $11(9 \%)$ fusariosis correspondiente a una incidencia de 0,004 por 1.000 egresos, menos frecuentes alternariosis siete casos $(6 \%)$, otros filamentosos cuatro (3\%) (Tabla 1) y 16 (13\%) casos en los que no se identificó agente fúngico específico.

La edad de los pacientes fue $50 \pm 16$ años, correspondiendo $57 \%$ de los casos a pacientes de género masculino. En relación a las patologías de base, $66,4 \%$ correspondían a enfermedad hematológica, $11,2 \%$ a TOS, $11,2 \%$ a enfermedad reumatológica y $11,2 \%$ a pacientes críticos $\mathrm{u}$ otras condiciones (Tabla 2). Ciento diecisiete $(93,6 \%)$ pacientes presentaban un factor de riesgo evidente siendo el más frecuente neutropenia en 56 pacientes $(44,8 \%)$, seguido de uso de terapia corticoesteroidal en $27(21,6 \%)$ y tratamiento inmunosupresor en $17(13,6 \%)$.

Clinicamente, $70(56 \%)$ pacientes manifestaron síntomas respiratorios bajos, $42(33,6 \%)$ síntomas rinosinusales, siete $(5,6 \%)$ lesiones de piel y tejidos blandos, dos $(1,6 \%)$ síntomas osteo-articulares, dos $(1,6 \%)$ abdominales y en uno $(0,8 \%)$ síntomas de compromiso del SNC.

Según criterios diagnósticos EORTC/MSG, los episodios incluidos clasificaron como EFI probada en 60 (48\%), probable en $51(40,8 \%)$ y posible en $14(11,2 \%)$ casos. El diagnóstico de EFI probada se basó en su totalidad en cultivos desde un tejido o cavidad estéril, principalmente tejido rinosinusal en 38 pacientes $(63,3 \%)$, tejido pulmonar o pleural en $13(21,7 \%)$, nódulos o lesiones cutáneas en siete $(11,7 \%)$ y tejido osteoarticular en dos $(3,3 \%)$. 
El diagnóstico de EFI probable se basó, en su criterio micológico, en la presencia de un cultivo o tinción de sitio no estéril en 16 pacientes $(31,4 \%)$, la presencia de GM positivo (sérico o en LBA) en 10 pacientes $(19,6 \%)$ y la presencia de ambos, cultivo y GM positivo, en 25 pacientes $(49 \%)$.

De los 67 casos de aspergilosis, en 51 pacientes $(76,1 \%)$ de los episodios se realizó al menos una medición de GM sérico, resultando positivo (IO 0,5) en 34 casos $(66,7 \%)$. En 26 pacientes $(38,8 \%)$ se realizó LBA, incluyéndose medición de GM en nueve, que resultó positivo (IO 1$)$ en ocho $(88,9 \%)$.

Todos los pacientes recibieron terapia antifúngica. Se indicó monoterapia en 103 pacientes $(82,4 \%)$ y terapia combinada en 22 pacientes $(17,6 \%)$, siendo voriconazol el antifúngico más frecuentemente prescrito en $56 \%$ de los episodios, seguido de anfotericina liposomal en $37 \%$, menos frecuentemente se utilizó: caspofungina 3\%, posaconazol $2 \%$, anfotericina desoxicolato e itraconazol en $1 \%$. Las terapias antifúngicas combinadas utilizadas en algún momento del tratamiento fueron voriconazol más anfotericina liposomal en $16(12,8 \%)$ pacientes, caspofungina con anfotericina liposomal en tres $(2,4 \%)$, voriconazol con caspofungina en uno $(0,8 \%)$, posaconazol con anfotericina liposomal en uno $(0,8 \%)$ paciente y voriconazol más caspofungina y anfotericina liposomal en uno $(0,8 \%)$ paciente. Respecto a defocación quirúrgica, ésta se realizó en $18(90 \%)$ de los casos de mucormicosis.

La mortalidad global a 30 días fue de $42 \%$.

\section{Comparación de períodos 2005-2009 vs 2010-2015}

Al comparar el número de episodios de EFI por hongos filamentosos, se identificaron 45 en 122.798 egresos en el períodos 2005-2009 vs 80 en 145.390 egresos en el período 2010-2015, correspondiendo a una incidencia de 0,37 por 1.000 egresos vs 0,55 por 1.000 egresos respectivamente $(p=0,0266)$. En ambos períodos la etiología más frecuente fue aspergilosis, seguido por mucormicosis y fusariosis. Las características generales de los episodios, categoría diagnóstica, condición de base se muestran en Tabla 3. Se debe destacar una tendencia a una menor mortalidad en el segundo período (49\% vs 39\%; $\mathrm{p}=\mathrm{NS}$ ).

\section{Características de EFI por condición de base de pacientes}

Del total de 125 casos, hubo $83(66,4 \%)$ en pacientes hemato-oncológicos y $42(33,6 \%)$ en no-hemato-oncológicos. En la Tabla 4 se comparan las características de ambos grupos. La edad del grupo hemato-oncológico fue relativamente menor así como la proporción de género masculino. Se hace evidente la ausencia de factor neutropenia en los casos de pacientes no-hematooncológicos, y al contrario, el alto número de pacientes con corticoesteroides o inmunosupresores en este grupo.
Tabla 2. Característcas generales de los 125 episodios de EFI identificados en el período de estudio

\begin{tabular}{lc}
\hline Características generales & $\mathbf{n}(\%)$ \\
\hline Edad, años (promedio \pm DS) & $50 \pm 16$ \\
\hline Género masculino & $71(57)$ \\
& \\
Hemato-oncológicos & $83(66,4)$ \\
- Leucemia mieloide aguda & $36(28,8)$ \\
- Leucemia linfocítica aguda & $17(13,6)$ \\
- Trasplante de precursores hematopoyéticos & $15(12)$ \\
- Linfoma no-Hodgkin & $6(4,8)$ \\
- Leucemia linfocítica cronica & $4(3,2)$ \\
- Otras condiciones & $5(4)$ \\
& $14(11,2)$ \\
Trasplante de órganos sólidos & $7(5,6)$ \\
- Hígado & $4(3,2)$ \\
- Corazón & $2(1,6)$ \\
- Riñón & $1(0,8)$ \\
- Intestino & $14(11,2)$ \\
& $8(6,4)$ \\
Reumatológicos & $4(3,2)$ \\
- Vasculitis & $2(1,6)$ \\
- Enfermedades sistémicas & $14(11,2)$ \\
- Artritis reumatoide & $3(2,4)$ \\
Pacientes críticos y otros & $3(2,4)$ \\
- Insuficiencia hepática & \\
- Insuficiencia renal y terapia de reemplazo renal & $8(6,4)$ \\
- Secuelas pulmonares/EPOC, enfermedad inflamatoria intestinal, & \\
cetoacidosis diabética, > 7 días en UCl, VIH &
\end{tabular}

Tabla 3. Comparación de episodios de EFI por filamentosos entre período 2005-2009 vs 2010-2015

\begin{tabular}{|c|c|c|c|}
\hline & $\begin{array}{l}2005-2009 \\
n=45(\%)\end{array}$ & $\begin{array}{l}2010-2015 \\
n=80(\%)\end{array}$ & Valor $\mathrm{p}$ \\
\hline Incidencia por 1.000 egresos & 0,37 & 0,55 & 0,0266 \\
\hline Edad, años (promedio \pm DS) & $49 \pm 17$ & $51 \pm 16$ & 0,2139 \\
\hline Género masculino & $27(60)$ & $41(51,3)$ & 0,193 \\
\hline Categoría diagnóstica EORTC probada + probable & $42(93,3)$ & $69(86,3)$ & 0,1193 \\
\hline $\begin{array}{ll}\text { Etiología episodio de EFI } \\
\text { - } & \text { Aspergilosis } \\
\text { - } & \text { Mucormicosis } \\
\text { - } & \text { Fusariosis } \\
\text { - } & \text { Otros filamentosos } \\
\text { - } & \text { EFI con cultivo negativo }\end{array}$ & $\begin{array}{l}26(57,8) \\
9(20) \\
2(4,4) \\
4(8,9) \\
4(8,9)\end{array}$ & $\begin{array}{l}41(51,3) \\
11(13,6) \\
9(11,3) \\
7(8,8) \\
12(15)\end{array}$ & $\begin{array}{l}0,2257 \\
0,1908 \\
- \\
- \\
-\end{array}$ \\
\hline $\begin{array}{l}\text { Condición de base } \\
\text { - } \quad \text { Hemato-oncológicos } \\
\text { - } \quad \text { TOS } \\
\text { - } \quad \text { Enfermedad reumatológica } \\
\text { Otras condiciones* }\end{array}$ & $\begin{array}{c}26(57,8) \\
10(22,2) \\
4(8,9) \\
5(11,1)\end{array}$ & $\begin{array}{c}57(71,3) \\
4(5) \\
10(12,5) \\
9(11,3)\end{array}$ & $\begin{array}{c}0,007 \\
0,0038 \\
0,1974 \\
0,5\end{array}$ \\
\hline Tratamiento asociado & $7(15,6)$ & $15(18,8)$ & 0,3373 \\
\hline Mortalidad a 30 días & $22(48,9)$ & $31(38,8)$ & 0,1389 \\
\hline
\end{tabular}




\begin{tabular}{|c|c|c|c|}
\hline & $\begin{array}{c}\text { Hemato- } \\
\text { oncológicos } \\
n=83(\%)\end{array}$ & $\begin{array}{c}\text { No hemato- } \\
\text { oncológicos } \\
n=42(\%)\end{array}$ & Valor $p$ \\
\hline Edad, años (promedio \pm DS) & $47 \pm 16$ & $55 \pm 16$ & 0,0047 \\
\hline Género masculino & $52(62,6)$ & $19(45,2)$ & 0,08 \\
\hline $\begin{array}{l}\text { Factores de riesgo } \\
\text { - Neutropenia } \\
\text { - Corticoesteroides } \\
\text { - Inmunosupresores } \\
\text { - Anticuerpos monoclonales }\end{array}$ & $\begin{aligned} 56 & (67,5) \\
6 & (7,2) \\
5 & (6) \\
0 & (0)\end{aligned}$ & $\begin{array}{c}0(0) \\
21(50) \\
12(28,6) \\
1(2,4)\end{array}$ & $\begin{array}{l}0,0000 \\
0,00001 \\
0,00001 \\
0,05\end{array}$ \\
\hline Categoría EORTC probada + probable & $71(85,5)$ & $40(95,2)$ & 0,06 \\
\hline $\begin{array}{l}\text { Etiología episodio de EFI } \\
\text { - Aspergilosis } \\
\text { - Mucormicosis } \\
\text { - Fusariosis } \\
\text { - Otros filamentosos } \\
\text { - No especificados }\end{array}$ & $\begin{array}{r}38(45,8) \\
15(18,1) \\
9(10,8) \\
8(9,6) \\
14(16,9)\end{array}$ & $\begin{aligned} & 29(69) \\
& 5(11,9) \\
& 2(4,8) \\
& 10(23,8) \\
& 2(4,8)\end{aligned}$ & $\begin{array}{l}0,007 \\
0,19 \\
0,133 \\
0,01 \\
0,029\end{array}$ \\
\hline Tratamiento asociado & $16(19,3)$ & $6(14,3)$ & 0,24 \\
\hline Mortalidad a 30 días & $34(41)$ & $19(45,2)$ & 0,33 \\
\hline
\end{tabular}

Destaca la significativa mayor frecuencia de aspergilosis en pacientes no-hemato-oncológicos y de fusariosis en hemato-oncológicos, aunque sin diferencia estadísticamente significativa, así como la mayor cantidad de EFI no especificada en este grupo. No hubo diferencias en la terapia ni en el desenlace de los episodios al comparar ambos grupos.

\section{EFI por Aspergillus spp. y no-Aspergillus spp.}

En los casos de aspergilosis, la edad de los pacientes fue $51 \pm 18$ años, correspondiendo en $55 \%$ de los casos a pacientes de sexo masculino, mientras que en las EFI por otros filamentosos, la edad fue de algo menor en fusariosis: $46 \pm 15$ años. La incidencia fue más alta en EFI por Aspergillus spp. 0,25 vs 0,16 por 1.000 egresos de las EFI por filamentosos no-Aspergillus.

La EFI fue clasificada según criterios EORTC/MSG como probada/probable en 61 (61\%) entre los casos de aspergilosis mientras que $100 \%$ de los casos no-Aspergillus fueron categoría probada/probable.

En EFI por Aspergillus spp., 88\% de los pacientes presentó algún factor de riesgo, principalmente neutropenia $(62 \%)$, uso de corticoterapia $(22 \%)$ y tratamiento inmunosupresor $(6 \%)$.

Respecto a co-morbilidades, en $100 \%$ de los casos existía una enfermedad de base; para los casos de aspergilosis, las principales fueron enfermedad hematológica: $57 \%$ (leucemias agudas $31 \%$, linfomas $10 \%$, leucemias crónicas $4 \%$, otros $13 \%$ ), enfermedad reumatológica:
$18 \%$ (vasculitis $10 \%$, enfermedades del tejido conectivo y artritis reumatoide $4 \%$ ), TOS: $13 \%$ (hígado $7 \%$, corazón y riñón 3\%) y otras condiciones: $10 \%$ (insuficiencia hepática $4 \%$, VIH 3\% y $1 \%$ secuelas pulmonares, enfermedad inflamatoria intestinal y terapia de reemplazo renal). En cambio para las EFI por filamentosos no-Aspergillus, fueron enfermedad hematológica: 74\% (leucemias agudas $45 \%$, leucemias crónicas $7 \%$, linfomas $5 \%$, aplasia medular $5 \%$, otros $11 \%$ ), otras condiciones: $16 \%$ (cetoacidosis diabética $7 \%$, enfermedad renal crónica $5 \%$, paciente crítico y enfermedad del tejido conectivo $2 \%$ cada uno) y TOS: $10 \%$ (corazón $5 \%$, hígado $2 \%$, intestino $2 \%$ ).

Respecto a la localización la EFI por Aspergillus, esta afectó principalmente al tejido pulmonar (73\%) y rinosinusal $(17 \%)$, en cambio la EFI por filamentosos no-Aspergillus comprometió principalmente al tejido rinosinusal (64\%), pulmonar (17\%) y cutáneo (12\%).

En $54(81 \%)$ casos de aspergilosis el tratamiento antifúngico fue monoterapia: voriconazol en $75 \%$ de los casos, anfotericina liposomal en $15 \%$, caspofungina en $6 \%$ y posaconazol, anfotericina-B desoxicolato e itraconazol en $1 \%$, y en $13(19 \%)$ pacientes terapia combinada: voriconazol con anfotericina liposomal en nueve pacientes (69\%), caspofungina con anfotericina liposomal en tres pacientes $(23 \%)$ y voriconazol con caspofungina en un paciente (8\%). En cambio, en las EFI por filamentosos no-Aspergillus se indicó tratamiento monoterapia en 36 pacientes (86\%) anfotericina liposomal en 79\%, voriconazol en $19 \%$ y posaconazol en $2 \%$ y terapia combinada en seis pacientes $(14 \%)$ voriconazol con anfotericina liposomal en siete pacientes $(78 \%)$, posaconazol con anfotericina liposomal en un paciente (14\%) y voriconazol con caspofungina con anfotericina liposomal en un paciente $(14 \%)$.

En $90 \%$ de los pacientes con mucormicosis se realizó manejo quirúrgico.

La mortalidad a 30 días fue de $39 \%$ en aspergilosis vs $57 \%$ en EFI por filamentosos no-Aspergillus, siendo la más alta la de mucormicosis: $70 \%(\mathrm{p}=0,003)$.

En la Tabla 5 se comparan las características generales, clínicas, terapia y resultados de las tres EFI por filamentosos más frecuentes identificadas en este estudio.

\section{EFI en pacientes inmunocomprometidos vs aparentemente inmunocompetentes}

Al comparar los 14 casos en pacientes aparentemente inmunocompetentes (estadía > 7 días en UCI, secuela pulmonar, EPOC, insuficiencia hepática, enfermedad renal crónica y terapia de reemplazo renal) con los 111 inmunocomprometidos (neoplasia hematológica, neoplasia de órganos sólidos, TPH, TOS, enfermedad reumatológica, enfermedad inflamatoria intestinal), no se identificaron diferencias significativas en edad, género ni categoría diagnóstica del episodio. En ambos grupos aspergilosis 


\begin{tabular}{|c|c|c|c|}
\hline & $\begin{array}{c}\text { Aspergilosis } \\
\mathrm{n}=67(\%)\end{array}$ & $\begin{array}{c}\text { Mucormicosis } \\
\mathrm{n}=20(\%)\end{array}$ & $\begin{array}{l}\text { Fusariosis } \\
\mathrm{n}=11(\%)\end{array}$ \\
\hline Edad, años (promedio \pm DS) & $51 \pm 18$ & $51 \pm 15$ & $46 \pm 15$ \\
\hline Género masculino & $37(55,2)$ & $10(50)$ & $6(54,5)$ \\
\hline $\begin{array}{l}\text { Patología de base } \\
\text { - Hemato-oncológicos } \\
\text { - TOS } \\
\text { - Reumatológicos } \\
\text { - Otras condiciones }\end{array}$ & $\begin{aligned} 38 & (56,7) \\
9 & (13,4) \\
12 & (17,9) \\
8 & (11,9) *\end{aligned}$ & $\begin{array}{l}15(75) \\
2(10) \\
1(5) \\
2(10) * *\end{array}$ & $\begin{array}{l}9(81,2) \\
0 \\
0 \\
2(18,2) * * *\end{array}$ \\
\hline $\begin{array}{l}\text { Factores de riesgo } \\
\text { - Neutropenia }(<500) \\
\text { - Corticoesteroides } \\
\text { - Inmunosupresores } \\
\text { - Trasplante de precursor hematopoyético }\end{array}$ & $\begin{array}{r}25(37,3) \\
19(28,4) \\
15(22,4) \\
5(7,5)\end{array}$ & $\begin{array}{l}9(45) \\
3(15) \\
2(10) \\
5(25)\end{array}$ & $\begin{array}{ll}6 & (54,5) \\
1 & (9,1) \\
1 & (9,1) \\
1 & (9,1)\end{array}$ \\
\hline $\begin{array}{l}\text { Localización } \\
\text { - Pulmonar } \\
\text { - Rinosinusal } \\
\text { - Cerebral } \\
\text { - Cutáneo } \\
\text { - Abdominal } \\
\text { - Osteoarticular }\end{array}$ & $\begin{aligned} 46 & (68,7) \\
13 & (19,4) \\
1 & (1,5) \\
2 & (3) \\
1 & (1,5) \\
& -\end{aligned}$ & $\begin{array}{c}3(15) \\
17(85)^{\wedge} \\
- \\
- \\
- \\
-\end{array}$ & $\begin{array}{cc}1 & (9,1) \\
3 & (27,3) \\
& - \\
5 & (45,5) \\
& - \\
2 & (18,2)\end{array}$ \\
\hline Terapia antifúngica combinada & $13(19,4)$ & $2(10)$ & $4(36,4)$ \\
\hline Mortalidad a 30 días & $2(3)$ & $14(70)^{\wedge} \wedge$ & $3(27,3)$ \\
\hline
\end{tabular}

fue la EFI más frecuente (45,5\% vs 54,4\%; $\mathrm{p}=\mathrm{NS}$ ), seguida por mucormicosis $(18,2$ vs $15,8 \%$; p $=\mathrm{NS})$ siendo a localización pulmonar más frecuente en ambos grupos (45,5 vs 57\%; $\mathrm{p}=\mathrm{NS})$. Se debe destacar que hubo mayor mortalidad en inmunocompetentes comparada con inmunocomprometidos (54 vs 41,2\%; $\mathrm{p}=\mathrm{NS}$ ).

\section{Discusión}

Este estudio de EFI por hongos filamentosos, el con mayor número de casos publicado en Chile, demuestra una incidencia de 0,47 por 1.000 egresos en el período 2005 2015 en un hospital terciario de referencia en Santiago, confirmando que aspergilosis es la EFI por filamentosos más frecuente en nuestro medio, seguida de agentes de mucormicosis y luego fusariosis.

Es interesante destacar que esta incidencia es muy similar a la de candidemia publicada en nuestro centro de 0,4 por 1.000 egresos en el período 2000 a $2013^{17}$, y corresponde a la misma cifra de incidencia de candidemia de 0,47 por 1.000 egresos, publicada recientemente en un estudio prospectivo que incluyó 26 hospitales a lo largo de Chile entre 2013 y $2017^{18}$. Sin duda, la frecuencia de
EFI por filamentosos demostrada en el presente estudio está influida fuertemente por el número de pacientes inmunocomprometidos tratados en nuestro centro, tanto hemato-oncológicos como receptores de trasplantes. Por el momento, no contamos con resultados de un estudio epidemiológico nacional que dé cuenta de las EFI por hongos filamentosos, a fin de conocer las características a nivel país. No es posible señalar cuánto pueden representar estos datos la realidad epidemiológica de otros hospitales de Chile, y solo podrá ser respondido a traves de un estudio prospectivo multicéntrico. Pueden existir algunas diferencias con otros centros, tanto en la misma ciudad de Santiago como de otras regiones, que pueden explicarse por tratarse el nuestro de un hospital de referencia para manejo de patología oncológica o trasplantes. Otros factores que pudieran influir en los resultados son: sus políticas de uso de antifúngicos, en particular profilaxis, disponibilidad de habitaciones de ambiente protegido para los pacientes de mayor riesgo, política de cuidado del ambiente hospitalario en relación a construcciones ${ }^{19}$, técnicas diagnósticas disponibles, así como las condiciones ambientales fuera del hospital tales como las variaciones climáticas, de humedad, vegetación, ventilación, carga de pólenes, tipos de suelo, 
nivel de desarrollo de la agroindustria, y eventualmente contaminación del aire ${ }^{7}$. Pero se puede plantear como hipótesis que en nuestro país, al menos en centros de referencia de características semejantes al nuestro, la incidencia de hongos filamentosos podría ser cercana a la aquí presentada.

Otro resultado a destacar, es que se confirma que en nuestro centro aspergilosis es la EFI por hongos filamentosos más frecuentemente identificada en todos los grupos de pacientes, lo que coincide con la mayoría de las publicaciones nacionales ${ }^{12-14}$ e internacionales ${ }^{1-3,20-24}$ de epidemiología de EFI por filamentosos. Sin embargo, es interesante hacer notar que en Brasil Fusarium spp. es el hongo filamentoso más frecuente en el subgrupo de $\mathrm{TPH}^{25}$ y en India los agentes de mucormicosis ocupan un lugar relevante en la frecuencia de infecciones por hongos filamentosos, explicado por condiciones ambientales y por un alto número de pacientes diabéticos mal contolados ${ }^{26}$.

El incremento de hongos filamentosos no-Aspergillus también ha sido reportada en la vigilancia de infecciones fúngicas en receptores de trasplantes TRANSNET, con incremento de la incidencia de mucormicosis de $0,08 \%$ a $0,69 \%$ en el período de 2001 a $2006^{27}$. En nuestra serie siguen en frecuencia agentes de mucormicosis y luego Fusarium spp., tal como se había documentado en publicaciones anteriores ${ }^{13,16}$. Lo que difiere con otras series, en Estados Unidos de América y algunos países de Europa, en que Fusarium spp. ha sido reportado en el segundo lugar de frecuencia en receptores de TPH y $\mathrm{TOS}^{3,23,34}$; en cambio, en Australia ha sido Scedosporium spp. ${ }^{3,28}$.

Un aspecto a destacar es la mayor tendencia a incrementar de las EFI no-Aspergillus, al punto de que en los últimos tres años de nuestra serie son más frecuentes que aspergilosis. Esto podría estar explicado por mayor uso de voriconazol con la consecuente emergencia de EFI por no-Aspergillus, diagnóstico más exhaustivo con mayor frecuencia de estudio invasor y pacientes más intensamente inmunocomprometidos; sin embargo, no es posible excluir otras variables dependientes del ambiente que puedan estar influyendo en esta variación. Sin duda será importante mantener una vigilancia prospectiva de las EFI por hongos filamentosos en nuestro centro, así como replicar en otros hospitales, para reconocer si se trata de un hecho puntual o que se repite a lo largo del país.

Respecto a las especies de Aspergillus, la más frecuente fue $A$. fumigatus, seguida más abajo de A. flavus, $A$. terreus y muy poco frecuente $A$. niger. En la literatura científica internacional también se describe $A$. fumigatus como el más frecuente, existiendo diferencias en la frecuencia de las siguientes especies, A. flavus o A. terreus como el segundo o tercero en frecuencia ${ }^{29}$. En una revisión de Chakrabarti y cols., que compara aspergilosis en países desarrollados con aquellos en desarrollo, los autores se- ñalan que $A$. fumigatus es el más frecuente, pero también destaca la importancia relativa de $A$. flavus como segundo agente en países en desarrollo, en especial en infecciones sino-orbitales y oculares ${ }^{30}$. En nuestro caso, si bien $A$. flavus es el siguiente en frecuencia, se encuentra junto a A. terreus. Una de las hipótesis planteadas por Chakrabarti y cols., sería la mayor frecuencia de contaminación del ambiente con A. flavus. Sería de interes contrastar especies de Aspergillus ambientales en nuestro medio con la frecuencia identificada en pacientes.

En nuestros resultados se debe destacar la baja frecuencia de $A$. terreus; este dato es particularmente útil ya que impacta en las opciones de terapia, por su conocida resistencia intrínseca a anfotericina.

Se debe señalar que hubo varios casos en que no se logró determinar la especie, tal como la serie de Neofitos y cols., en que en 53\% de los Aspergillus no se identificó la especie ${ }^{5}$. De igual forma, es importante destacar que la identificación de especie utilizada en el laboratorio se basa principalmente en la observación de las características macro y microscópicas de los hongos, no siendo posible, en muchos casos, identificar correctamente a las distintas especies pertenecientes a un complejo. De esta forma, si bien no se identificaron especies como $A$. nidulans, $A$. lentulus, $A$. udagawae, $A$. viridinutans, entre otras $^{31}$, esto puede deberse a problemas metodológicos. La correcta identificación a nivel de especie requiere del uso de técnicas moleculares. La secuenciación de las regiones ITS1 e ITS2 asociado a la secuenciación de los genes de la $\beta$-tubulina o calmodulina permitirían llegar a la identificación final; sin embargo, este algoritmo es complejo y no está disponible en la mayoría de los laboratorios de microbiología clínicos ${ }^{32}$. Finalmente, se ha reportado la identificación de $A$. lentulus mediante espectrometría de masas (MALDI-TOF); faltan, sin embargo, mayores estudios para recomendar su uso ${ }^{33}$.

Una de las limitaciones del estudio es que no contamos con información de susceptibilidad a los antifúngicos en las cepas identificadas, ya que al tiempo de su presentación no se contaba con la técnica correspondiente. A nuestro conocimiento, no disponemos de información nacional de los patrones de resistencia de los hongos filamentosos, por lo que urge contar con estos datos, especialmente porque internacionalmente se ha reportado de manera creciente resistencia de Aspergillus spp. a azoles que se ha relacionado al uso de antifúngicos en la industria agroalimentaria, identificándose mutaciones que se asocian a esta resistencia, la que ha sido reportada en todos los continentes ${ }^{34}$. La mayor parte de los datos provienen de Europa y Asia, en que se describen en algunos centros de referencia resistencia de $A$. fumigatus a azoles de hasta $30 \%{ }^{35}$, con impacto en el desenlace de las infecciones. De hecho, la resistencia de $A$. fumigatus a azoles lleva a $21 \%$ incremento de mortalidad ${ }^{36}$. En nuestro continente los 
estudios realizados no han logrado identificar frecuencias relevantes en las mutaciones asociadas a resistencia que hagan plantear cambios en la práctica clínica ${ }^{37}$, pero sin duda, es un tópico en evolución.

Las recientes guías de manejo de aspergilosis de la ESCMID-ECMM-ERS sugieren que, si la vigilancia de resistencia a azoles de $A$. fumigatus es mayor de $10 \%$, se debe cambiar el enfrentamiento inicial, ya sea asociando equinocandinas o prefiriendo anfotericina liposomal ${ }^{38}$. Es indudable que se requieren estudios dirigidos a conocer la susceptibilidad de hongos filamentosos en nuestro país, en particular Aspergillus spp., lo que es muy relevante para el diseño de guías clínicas de enfrentamiento inicial o para los casos en que no se logra la identificación microbiológica de Aspergillus spp. A su vez, contar con el estudio rutinario de susceptibilidad a antifúgicos frente al caso puntual es crítico para la toma de decisiones en los casos con infección documentada.

La mortalidad en nuestra serie es comparable a lo publicado en series de países desarrollados; pensamos que el diagnóstco oportuno facilitado por las herramientas diagnósticas disponibles, el acceso expedito a antifúngicos y el manejo quirúrgico en los casos que lo ameritan, permiten llegar a estas cifras ${ }^{39}$, las que podrían aún mejorarse, a través de la estandarización de las prácticas, la definición de algoritmos de diagnósticos y de manejo.

Otro aspecto interesante de nuestro estudio son las EFI por hongos filamentosos en otros grupos de pacientes; por una parte, aquellos inmunocomprometidos por otras causas no hemato-oncológicos ni receptores de trasplantes, así como pacientes aparentemente inmunocompetentes. Entre éstos se presentaron pacientes en terapia con inmunosupresores por distintos motivos en los que no siempre se tiene presente los hongos filamentosos como etiología de sinusitis, neumonia u otras localizaciones ${ }^{40}$. Y en cuanto a los inmunocompetentes, si bien es un grupo menor respecto a los inmunocomprometidos, son pacientes que se describen cada vez con mayor frecuencia en la literatura médica más reciente ${ }^{41}$; son enfermos como aquellos con EPOC, secuelas pulmonares, daño hepático, falla renal con terapia de reemplazo renal, en los que aspergilosis es la EFI más frecuente, lo que pone en relevancia este tema y clínicamente obliga a estar muy atentos a la evolución de enfermos críticos y tener especial cautela en la interpretación de cultivos de la vía aérea que demuestren hongos filamentosos.
En conclusión, este estudio aporta información epidemiológica de EFI causada por hongos filamentosos de un centro con alta actividad en pacientes hemato-oncológicos $\mathrm{y}$ receptores de trasplantes, en que se demuestra un incremento progresivo de su frecuencia, en especial hongos filamentosos no-Aspergillus en los útimos años, pero que aspergilosis lidera este tipo de infecciones en los diferentes grupos de pacientes. Se requiere avanzar en la investigación epidemiológica nacional a fin de conformar un panorama completo de este tipo de infeciones para la construcción de guías nacionales de diagnóstico y terapia basadas en datos propios y avanzar en la investigación de susceptibilidad a antifúngicos y vigilancia de infecciones por hongos filamentosos en grupos de pacientes emergentes.

\section{Resumen}

Introducción: La enfermedad fúngica invasora (EFI) por hongos filamentosos es cada vez más frecuente. Objetivo: Estudiar la epidemiología de la EFI en adultos hospitalizados en nuestro centro. Metodología: Estudio retrospectivo de pacientes adultos de un hospital universitario en Santiago, Chile, con EFI por hongos filamentosos entre enero de 2005 y diciembre de 2015. Resultados: Se identificaron 125 episodios, siendo $48 \%$ categoria probada, $40 \%$ probable y $11 \%$ posible según criterios EORTC/ MSG, incidencia global 0,47 x 1.000 egresos, 57\% pacientes masculinos y edad de $50 \pm 16$ años. El 66,4\% tenía patología hematológica, $11,2 \%$ trasplante de órgano sólido, $11,2 \%$ enfermedad reumatológica, $11,2 \%$ otra condición. Los factores de riesgo fueron neutropenia $44 \%$, corticoterapia $21 \%$, inmunosupresores $13 \%$. Los hongos más frecuentemente identificados fueron Aspergillus spp (53,6\%), Mucorales (16\%), Fusarium spp (8,8\%), Alternaria spp (5,6\%), otros filamentosos (3,2\%). Todos recibieron antifúngicos, $82 \%$ monoterapia, $18 \%$ terapia combinada, hubo defocación quirúrgica en $90 \%$ de mucormicosis. La mortalidad global fue $42 \%$. Al comparar 2005-2009 vs 2010-2015, hubo un aumento significativo de la incidencia y una tendencia a menor mortalidad en el segundo período. Conclusiones: Durante un período de 10 años, observamos un aumento de la incidencia de EFI por filamentosos, aspergilosis fue la etiología más frecuente y la mortalidad global fue $42 \%$. 


\section{Referencias bibliiográficas}

1.- Lemonovich T L. Mold infections in solid organ transplant recipients. Infect Dis Clin North Am. 2018; 32: 687-701. doi: 10.1016/j. idc.2018.04.006.

2.- Pagano L, Caira M, Candoni A, Offidani M, Fianchi L, Martino B, et al. The epidemiology of fungal infections in patients with hematologic malignancies: the SEIFEM-2004 study. Haematologica 2006; 91: 1068-75. PMID: 16885047.

3.- Douglas A P, Chen S C A, Slavin M A. Emerging infections caused by non-Aspergillus filamentous fungi. Clin Microbiol Infection 2016; 22: 670-80. doi: https://doi.org/10.1016/j. cmi.2016.01.011

4.- Ben-Ami R, Lewis R E, Kontoyiannis D P. Invasive mould infections in the setting of hematopoietic cell transplantation: current trends and new challenges. Curr Opin Infect Dis 2009; 22: 376-84. doi: 10.1097/ QCO.0b013e32832db9f3.

5.- Neofytos D, Hom D, Anaissie E, Steinbach W, Olyaei A, Fishman J, et al. Epidemiology and outcome of invasive fungal infection in adult hematopoietic stem cell transplant recipients: analysis of Multicenter Prospective Antifungal Therapy (PATH) Alliance registry. Clin Infect Dis. 2009; 48: 265-73. doi: 10.1086/595846.

6.- García-Ruiz J C, Amutio E, Ponton J. Invasive fungal infection in immunocompromised patients. Rev Iberoam Micol. 2004; 21: 55-62. PMID:15538828.

7.- Rabagliati R. Actualización en el diagnóstico y manejo de aspergilosis invasora en pacientes adultos. Rev Chilena Infectol. 2018; 35: 53144. doi: 10.4067/s0716-10182018000500531.

8.- Gamarra S, Chaves M S, Cabeza M S, Macedo D, Leonardelli F, Franco D, et al. Mucormycosis outbreak due to Rhizopus microsporus after arthroscopic anterior cruciate ligament reconstruction surgery evaluated by RAPD and MALDI-TOF Mass spectrometry. J Mycol Med. 2018; 28: 617-22. doi: 10.1016/j. mycmed.2018.09.002.

9.- Smith R M, Schaefer M K, Kainer M A, Wise M, Finks J, Duwve J, et al. Fungal infections associated with contaminated methylprednisolone injections. N Engl J Med. 2013; 369: 1598-609. doi: 10.1056/ NEJMoa1213978.

10.- Orsini M, Otaíza F, Vega P, Hederra L M, Pidal P, Salas V, et al. Brote de fungemias por Sarocladium kiliense en ocho hospitales públicos por contaminación intrínseca de ondansetrón intravenoso. Rev Chilena Infectol. 2018; 35: 363-70. doi: 10.4067/s071610182018000400363.

11.- De Pauw B, Walsh T J, Donnelly P, Stevens D A, Edwards J E, Calandra T, et al. Revised definitions of invasive fungal disease from the European Organization for Research and Treatment of Cancer/Invasive Fungal Infections Cooperative Group and the National Institute of Allergy and Infectious Diseases Mycoses Study Group (EORTC/MSG) Consensus Group. Clin Infect Dis 2008; 46: 1813-21.

doi: $10.1086 / 588660$.

12.- Álvarez Duarte E, Denning D W. Serious fungal infections in Chile. Eur J Clin Microbiol Infect Dis. 2017; 36: 983-6. doi: 10.1007/ s10096-017-2925-8.

13.- Rabagliati B R, Fuentes G, Guzmán A, Orellana E, Oporto J, Aedo I. Enfermedad fúngica invasora en pacientes hemato-oncológicos y receptores de trasplante de precursores hematopoyéticos bajo la perspectiva de los criterios diagnósticos EORTC/MSG. Rev Chil Infectol. 2009; 26: 212-9. doi: /S071610182009000400002 .

14.- Cruz R, Piontelli E. Enfermedad fúngica invasora en pacientes de cinco hospitales de la Región de Valparaíso, Chile. Rev Chilena Infectol. 2011; 28: 123-9. doi: /S071610182011000200004.

15.- Cruz R, Álvarez P, Provoste F, Ducasse K, González M, Wilson G, et al. Enfermedad fúngica invasora (EFI) por hongos filamentosos en la Región de Valparaíso, Chile, desde la implementación del diagnóstico rápido de laboratorio. Rev Chilena Infectol. 2015; 32: 221-4. doi: 10.4067/S071610182015000300012.

16.- Rabagliati R, Bertín P, Cerón I, Rojas H, Domínguez I, Vera Á, et al. Epidemiología de neutropenia febril en pacientes adultos con leucemia aguda y linfoma. Estudio de cohorte en hospitales público y privado de Santiago, Chile. Rev Chilena Infectol. 2014; 31:721-8. doi: $10.4067 / \mathrm{S} 0716-10182014000600013$.

17.- Siri L, Legarraga P, García P, González T, Rabagliati R. Cambios clínicos y epidemiológicos de candidemias en pacientes adultos desde 2000 a 2013. Rev Chilena Infectol. 2017; 34: 19-26. doi: 10.4067/S071610182017000100003.

18.- Santolaya M E, Thompson L, Benadof D, Tapia C, Legarraga P, Cortés C, et al. A prospective, multi-center study of Candida bloodstream infections in Chile. PLoS One. 2019; 14 : e0212924. doi: 10.1371/journal.pone.0212924.

19.- Rabagliati R, Catalán P, Rabello M, Ajenjo M C, Zubieta M. Variables del ambiente hospitalario que inciden en el riesgo de infecciones de pacientes con cáncer y receptores de trasplante de precursores hematopoyéticos: Diseño, procesos asistenciales, calidad microbiológica del aire y agua. Rev Chilena Infectol 2019; 36: 126-138. doi: 10.4067/S071610182019000200126.

20.- Kontoyiannis D P, Marr K A, Park B J, Alexander B D, Anaissie E J, Walsh T J.
Prospective surveillance for invasive fungal infections in hematopoietic stem cell transplant recipients, 2001e2006: overview of the transplant-associated infection surveillance network (TRANSNET) database. Clin Infect Dis 2010; 50: 1091e100. doi: 10.1086/651263.

21.- Girmenia C, Raiola A M, Piciocchi A, Algarotti A, Stanzani M, Cudillo L, et al. Incidence and outcome of invasive fungal diseases after allogeneic stem cell transplantation: a prospective study of the Gruppo Italiano Trapianto Midollo Osseo (GITMO). Biol Blood Marrow Transplant 2014; 20: 872-80. doi: 10.1016/j. bbmt.2014.03.004.

22.- Chamilos G, Luna M, Lewis R E, et al. Invasive fungal infections in patients with hematologic malignancies in a tertiary care cancer center: an autopsy study over a 15 -year period (1989-2003). Haematologica 2006; 91 : 986-9.

23.- Park B J, Pappas P G, Wannemuehler K A, Alexander B D, Anaisse E J, Andes D R, et al. Invasive non-Aspergillus mould infections in transplant recipients, United States, 2001e2006. Emerg Infect Dis 2011; 17: 1855-64. doi: 10.3201/eid1710.110087.

24.- Sun Y, Meng F, Han M, Zhang X, Yu L, Huang $\mathrm{H}$, et al. Epidemiology, management, and outcome of invasive fungal disease in patients undergoing hematopoietic stem cell transplantation in China: A multicenter prospective observational study. Biol Blood Marrow Transplant 2015; 21: 1117-26. doi: 10.1016/j.bbmt.2015.03.018.

25.- Nucci M, Garnica M, Gloria AB, Lehugeur DS, Dias VC, Palma LC, et al. Invasive fungal diseases in haematopoietic cell transplant recipients and in patients with acute myeloid leukaemia or myelodysplasia in Brazil. Clin Microbiol Infect 2013; 19: 745-51. doi: 10.1111/1469-0691.

26.- Chakrabarti A, Das A, Mandal J, Shivaprakash M R, George V K, Tarai B, et al. The rising trend of invasive mucormycosis in patients with uncontrolled diabetes mellitus. Med Mycol 2006; 44: 335-42. doi: 10.1080/13693780500464930.

27.- Park B J, Pappas P G, Wannemuehler K A, Alexander B D, Anaissie E J, Andes D R, et al. Invasive non-Aspergillus mold infections in transplant recipients, United States, 20012006. Emerg Infect Dis. 2011; 17: 1855-64. doi: 10.3201/eid1710.110087.

28.- Slavin M, van Hal S, Sorrell T C, Lee A, Marriot D J, Daveson K, et al. Invasive infections due to filamentous fungi other than Aspergillus: epidemiology and determinants of mortality. Clin Microbiol Infect 2015; 21. 490.e1e10. doi: 10.1016/j. cmi.2014.12.021. 
29.- Enoch D A, Yang H, Aliyu A H, Micallef C. The changing epidemiology of invasive fungal infections. Chapter 2: 17-65. In Thomas Lion (ed.), Human Fungal Pathogen Identification: Methods and Protocols, Methods in Molecular Biology, vol. 1508, DOI 10.1007/978-1-49396515-1_2.

30.- Chakrabarti A, Chatterjee S S, Das A, Shivaprakash M R. Invasive aspergillosis in developing countries. Medical Mycology 2011, 49: S35-S47. doi: 10.3109/13693786.2010.505206.

31.- Gautier M, Normand A-C, Ranque S. Previously unknown species of Aspergillus. Clin Microb Infect 2016; 22: 662-9. doi: 10.1016/j.cmi.2016.05.013.

32.- Samson R A, Visagie C M, Houbraken J, Hong $\mathrm{S}$ B, Hubka V, Klaassen C H, et al. Phylogeny, identification and nomenclature of the genus Aspergillus. Stud Mycol 2014; 78: 141-73. doi: 10.1016/j.simyco.2014.07.004.

33.- Vidal-Acuña M R, Ruiz-Pérez de Pipaón M, Torres-Sánchez M J, Aznar J. Identification of clinical isolates of Aspergillus, including cryptic species, by matrix assisted laser desorption ionization time-of-flight mass spectrometry (MALDI-TOF MS). Med Mycol 2018; 56 (7): 838-46. doi: 10.1093/mmy/ myx 115 .

34.- Gonçalves S S, Souza A C R, Chowdhary A, Meis J F, Colombo A L. Epidemiology and molecular mechanisms of antifungal resistance in Candida and Aspergillus. Mycoses 2016; 59: 198-219. doi: 10.1111/myc.12469.

35.- Lestrade P P, Meis J F, Arends J P, van der Beek M T, de Brauwer E, van Dijk K, et al. Diagnosis and management of aspergillosis in the Netherlands: a national survey. Mycoses. 2016; 59: 101-7. doi: 10.1111/myc. 12440.

36.- Resendiz Sharpe A, Lagrou K, Meis J F, Chowdhary A, Lockhart S R, Verweij P E, et al.Triazole resistance surveillance in Aspergillus fumigatus. Med Mycol. 2018; 56 (suppl 1): 83-92. doi: 10.1093/mmy/myx144.

37.- Negri C E, Gonçalves S S, Sousa A C P, Bergamasco M D, Martino M D V, QueirozTelles F, et al. Triazole resistance is still not emerging in Aspergillus fumigatus isolates causing invasive aspergillosis in Brazilian patients. Antimicrob Agents Chemother. 2017; 61 (11). pii: e00608-17. doi: 10.1128/ AAC.00608-17.

38.- Ullmann A J, Aguado J M, Arikan-Akdagli S, Denning D W, Groll A H, Lagrou K, et al. Diagnosis and management of Aspergillus diseases: executive summary of the 2017 ESCMID-ECMM-ERS guideline. Clin Microbiol Infect. 2018; 24 Suppl 1:e1-e38. doi: 10.1016/j.cmi.2018.01.002.

39.- Lamoth F, Calandra T. Early diagnosis of invasive mould infections and disease. $\mathrm{J}$ Antimicrob Chemother 2017; 72 (suppl_1): i19-i28. doi: 10.1093/jac/dkx030.

40.- Bassetti M, Peghin M, Vena A. Challenges and solution of invasive aspergillosis in nonneutropenic patients: A review. Infect Dis Ther. 2018; 7: 17-27. doi: 10.1007/s40121-017-01839.

41.- Bassetti M, Bouza E. Invasive mould infections in the ICU setting: complexities and solutions. J Antimicrob Chemother. 2017; 72 (suppl_1): i39-i47. doi: 10.1093/jac/dkx032. 